\title{
Combined Sublabial and Transconjunctival Approach for Huge Maxillary Fibrous Dysplasia
}

\author{
Han-Seul Na, MD, Ji-Hwan Park, MD, Sung-Dong Kim, MD, PhD and Kyu-Sup Cho, MD, PhD \\ Department of Otorhinolaryngology and Biomedical Research Institute, \\ Pusan National University School of Medicine, Pusan National University Hospital, Busan, Korea
}

\section{- ABSTRACT -}

Fibrous dysplasia (FD) is a benign progressive fibro-osseous lesion that frequently occurs in the craniofacial region. The maxilla is the most commonly involved facial bone, with facial asymmetry and esthetic problems being the usual complaints. The main treatment for maxillary FD is surgery. Surgical management strategies vary depending on the location, size, and severity of the lesion. Herein we describe a case of huge maxillary FD with severe facial deformity, which was completely removed by combined sublabial and transconjunctival approach. (J Clinical Otolaryngol 2021;32:149-152)

KEY WORDS: Fibrous dysplasia; Monostotic; Maxilla; Mouth; Conjunctiva.

\section{Introduction}

Fibrous dysplasia (FD) is a benign progressive fibro-osseous lesion that is characterized by a fibrous stroma and immature woven bone replacing normal bone. ${ }^{1)}$ The craniofacial bones are affected in most patients with polyostotic FD, and 15\%-30\% with monostotic FD. ${ }^{2}$ Patients with craniofacial FD most commonly experience painless, gradual skull and facial bone growth. ${ }^{3)}$ Although surgery is the treatment of choice for craniofacial FD, surgical treatment options can vary and depend on the patient's age, aggressiveness, extent, and site of the lesion. ${ }^{4)}$ This case report describes monostotic huge maxillary FD with severe facial deformity, which was completely removed by combined sublabial and transconjunctival approach.

\section{Case Report}

A 40-year-old male presented with gradually progressive left cheek swelling and nasal obstruction for 5 years (Fig. 1). He had no relevant medical history and no other rhinological or ophthalmological symptoms. The endoscopic examination revealed bulging of lateral nasal wall and nasal septum deviation to left side. A computed tomography (CT) of the paranasal sinuses showed $4.2 \times 3.3 \times 4.0 \mathrm{~cm}$ sized expansile enhancing mass with ground glass opacity and sclerotic margin at left maxillary sinus anterior wall. Furthermore, An enlarged left maxillary bone was observed (Fig. 2). These findings were consistent with a maxillary FD. Considering patient's age and postoperative morbidity, surgical access was gained via combined sublabial and transconjunctival approach under general anesthesia. After

Received: August 26, 2021 / Revised: September 3, 2021 / Accepted: September 10, 2021

Corresponding author: Kyu-Sup Cho, Department of Otorhinolaryngology and Biomedical Research Institute, Pusan National University School of Medicine, Pusan National University Hospital, Busan 49241, Korea Tel: +82-51-240-7824 Fax: +82-51-246-8668·E-mail: choks@pusan.ac.kr 


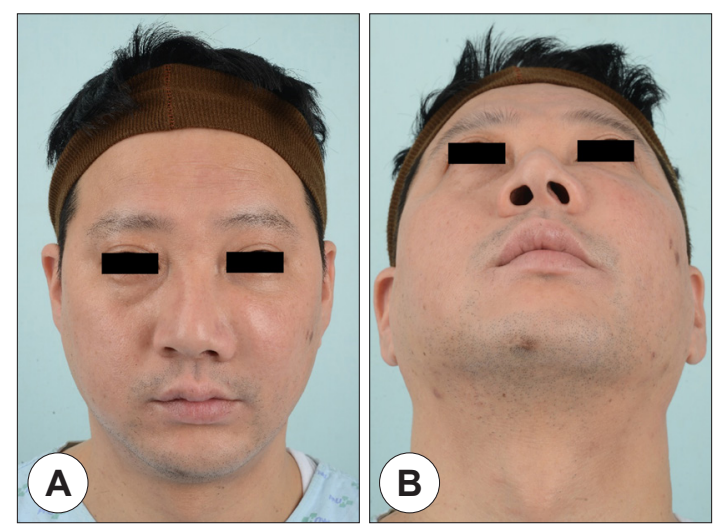

Fig. 1. Preoperative profiles. Frontal (A) and basal (B) views show left cheek swelling and facial asymmetry.

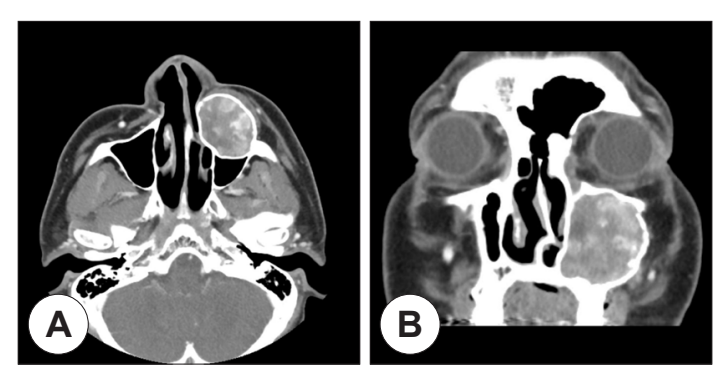

Fig. 2. Preoperative paranasal sinus computed tomography. A bulging enhancing lesion with ground glass opacity and sclerotic margin is shown at left maxillary sinus anterior wall in axial (A) and coronal (B) images. resection of the main mass, the remaining lesions were completely excised using grasping forceps and curved blade or burr of a microdebrider (Medtronic Xomed, Jacksonville, FL, USA) through visualization with a nasal endoscope. Reconstruction was accomplished using Medpor $\operatorname{Titan}^{\circledR}$ (Stryker, Kalamazoo, MI, USA) to cover the bone defect on orbital floor, orbital rim, and anterior wall of maxillary sinus. Histological examination showed curvilinear trabeculae of woven bone and fibroblastic stroma lacking prominent osteoblastic rimming (Fig. 3). These findings were consistent with FD. There were no specific complications postoperatively, and the patient was satisfied cosmetically (Fig. 4). Endoscopic examination and CT performed 12 months postoperatively showed no evidence of recurrence (Fig. 5).

\section{Discussion}

FD is a rare disease of unknown etiology which can involve single (monostotic) or multiple (polyostotic) bones. Craniofacial involvement mainly occurs in polystotic FD, but it also occurs in about $10 \%$ of patients with monostotic FD. The most common involvement
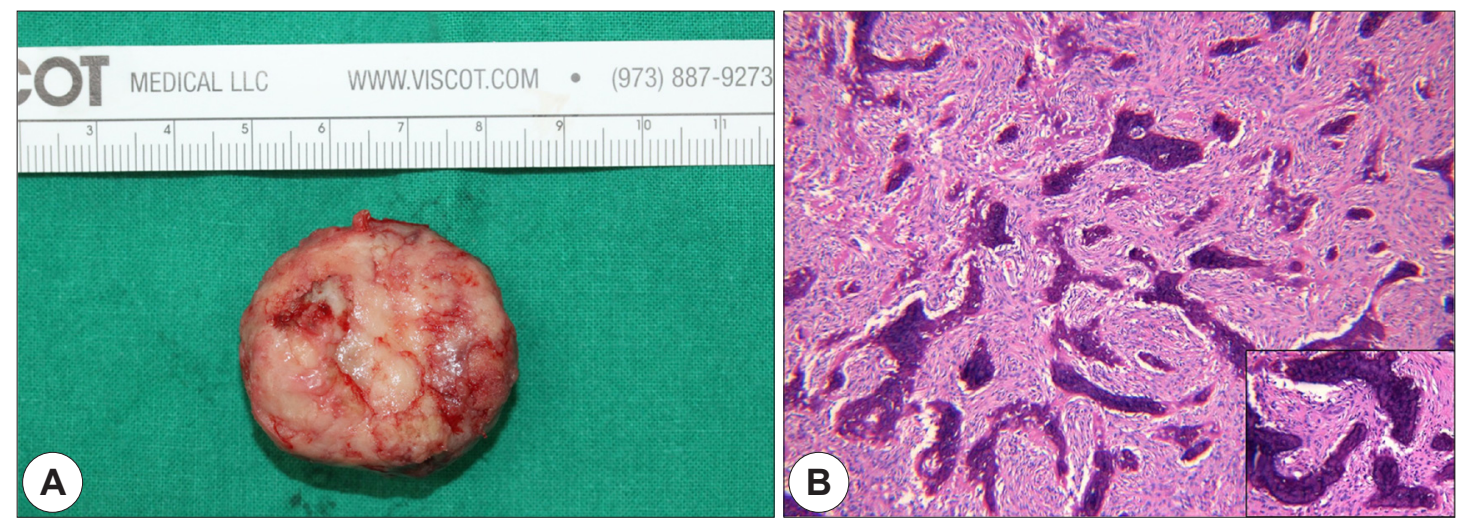

Fig. 3. Histopathologic findings of fibrous dysplasia. (A) The mass was removed via combined sublabial and transconjunctival approach and measured bulging $4.2 \times 3.3 \times 4.0 \mathrm{~cm}$. (B) The specimen section shows curvilinear trabeculae of woven bone and fibroblastic stroma $(\mathrm{H} \& \mathrm{E}, \times 100)$ and neoplastic bone lacking prominent osteoblastic rimming (inlet, $H \& E, \times 400$ ). 


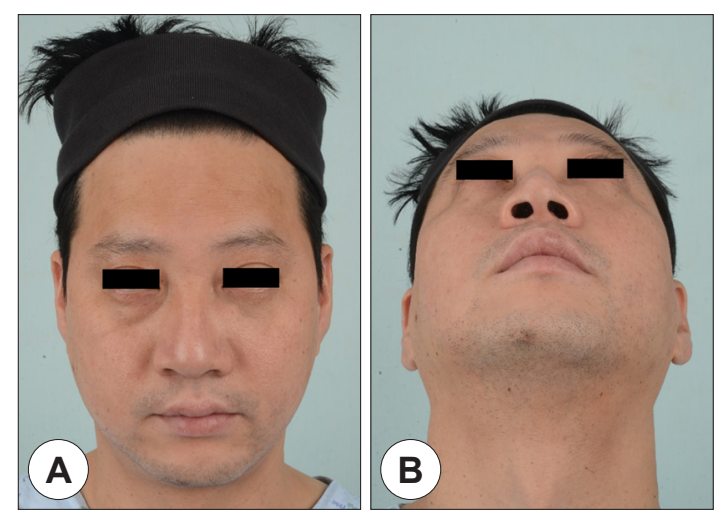

Fig. 4. Postoperative profiles. Frontal (A) and basal (B) views show satisfactory cosmetic outcome and favorable facial symmetry.
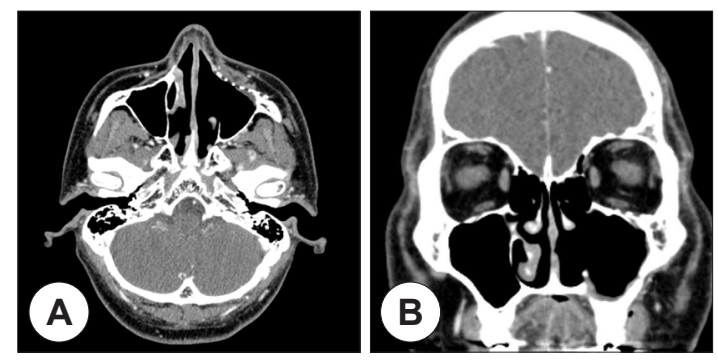

Fig. 5. Postoperative paranasal sinus computed tomography images at postoperative 1 year. There are no evidence of recurrence in axial (A) and coronal (B) images.

is in the skull bones, in descending order, are frontal, sphenoid, ethmoid, parietal, temporal, and occipital bones. ${ }^{5}$ In the facial region, FD is most commonly found in the maxillary bones. ${ }^{6}$

The clinical feature of craniofacial FD varies depending on their extent and location of lesions. Such symptoms include buccolingual expansion with jaw deformities, nasal congestion, facial pain and asymmetry. However, gradual and painless facial swelling was reported to be the most common presenting symptom. Often, FD can cause structural deformation of the orbit and auditory canal, which can lead to visual impairment or hearing loss, respectively. ${ }^{7}$

FD could be suspected when there are characteristic radiological findings. A typical finding of FD is an expansile and intramedullary lesion with a "groundglass appearance". ${ }^{8)}$ Because of FD's most specific findings on $\mathrm{CT}$, it should be performed first in patients with symptoms typical of FD, such as asymmetric facial swelling. On the other hand, MR images have limitations in evaluating bone structures. A typical microscopic finding of FD is the observation of irregularly shaped bony trabeculae with the background of loosely arranged fibrous stroma. The C- and S-shaped woven bone trabeculae with little osteoblastic rimming are thin and disconnected to each other, and this have been described as "chinese characters" 1,6)

The general principle of treatment for FD is surgical resection. The surgical approaches for craniofacial FD vary depending on the size, location, and extent of the lesion. ${ }^{1)}$ These include curettage, conservative contouring, partial resection, and total resection. For the surgical accesses of maxillary FD, various approaches such as the gingivobuccal or bicoronal approach, the midfacial degloving, and the Weber-Ferguson approaches have been implemented. ${ }^{9)}$ However, the Weber-Ferguson incision causes a large scar on the external skin of the face and midfacial degloving approach may have complications such as permanent infraorbital numbness and paresthesia, nasal cosmetic deformities, oronasal or oroantral fistula, and vestibular stenosis. In this case, a gingivobuccal approach alone was not sufficient as the FD had extended to the orbital rim. Through the combined subalabial and transconjunctival incisions, it was possible to access the mass from both directions, above and below, and huge maxillary FD was successfully removed. This technique ensured complete visualization of the infraorbital rim and wider exposure of the maxillary FD. There were no postoperative complications such as infraorbital numbness, ocular foreign body sensation and cosmetic complaints, and there was no recurrence at 1 year on follow-up CT. Therefore, this technique is well tolerated by patients and may be easily applicable to the maxillary FD. 


\section{Conclusion}

The combined sublabial and transconjunctival approach had a good surgical outcome because of excellent exposure of lesion, and there was no scar, so it was possible to obtain a satisfactory result cosmetically. Therefore, the combined sublabial and transconjunctival approach is a highly effective and safe technique with low morbidity for the treatment of maxillary FD.

\section{Acknowledgements}

Not applicable.

\section{Funding Information}

Not applicable.

\section{Conflict of Interest}

No potential conflict of interest relevant to this article was reported.

\section{ORCID}

Han-Seul Na, https://orcid.org/0000-0002-0118-3887 Ji-Hwan Park, https://orcid.org/0000-0001-9301-6957 Sung-Dong Kim, https://orcid.org/0000-0002-8436-5722 Kyu-Sup Cho, https://orcid.org/0000-0002-4381-6996

\section{Author Contribution}

Conceptualization: Cho KS.

Validation: Kim SD.

Investigation: Park JH, Na HS.
Writing - original draft: Na HS.

Writing - review \& editing: Cho KS, Kim SD, Park JH, Na HS.

\section{Ethics Approval}

This case was approved by the Institutional Review Board of Pusan National University Hospital (IRB number: 2101-018-099).

\section{References}

1) Wie CE, Hong SL, Mun SJ, Cho KS. Endoscopic marsupialization of frontoethmoid mucocele with underlying craniofacial fibrous dysplasia. J Craniofac Surg 2015;26(1):e73-4.

2) Panda NK, Parida PK, Sharma R, Jain A, Bapuraj JR. A clinicoradiologic analysis of symptomatic craniofacial fibro-osseous lesions. Otolaryngol Head Neck Surg 2007;136(6):92833.

3) Menon S, Venkatswamy S, Ramu V, Banu K, Ehtaih S, Kashyap VM. Craniofacial fibrous dysplasia: surgery and literature review. Ann Maxillofac Surg 2013;3(1):66-71.

4) Valentini V, Cassoni A, Marianetti TM, Terenzi V, Fadda MT, Iannetti G. Craniomaxillofacial fibrous dysplasia: conservative treatment or radical surgery? A retrospective study on 68 patients. Plast Reconstr Surg 2009;123(2):653-60.

5) DiCaprio MR, Enneking WF. Fibrous dysplasia: pathophysiology, evaluation, and treatment. J Bone Joint Surg Am 2005; 87(8):1848-64

6) MacDonald-Jankowski D. Fibrous dysplasia: a systematic review. Dentomaxillofac Radiol 2009;38(4):196-215.

7) Nambi GI, Jacob J, Gupta AK. Monofocal maxillary fibrous dysplasia with orbital, nasal and oral obstruction. J Plast Reconstr Aesthet Surg 2010;63(1):e16-8.

8) Burke AB, Collins MT, Boyce AM. Fibrous dysplasia of bone: craniofacial and dental implications. Oral Dis 2017;23 (6):697-708.

9) Kusano T, Hirabayashi S, Eguchi T, Sugawara Y. Treatment strategies for fibrous dysplasia. J Craniofac Surg 2009; 20(3):768-70. 